\title{
SOME RESULTS ON THE INDEPENDENCE POLYNOMIAL OF UNICYCLIC GRAPHS
}

\author{
Mohammad Reza Oboudi \\ Department of Mathematics, College of Sciences \\ Shiraz University, Shiraz, 71457-44776, Iran \\ and \\ School of Mathematics \\ Institute for Research in Fundamental Sciences (IPM) \\ P.O. Box 19395-5746, Tehran, Iran \\ e-mail: mr_oboudi@yahoo.com
}

\begin{abstract}
Let $G$ be a simple graph on $n$ vertices. An independent set in a graph is a set of pairwise non-adjacent vertices. The independence polynomial of $G$ is the polynomial $I(G, x)=\sum_{k=0}^{n} s(G, k) x^{k}$, where $s(G, k)$ is the number of independent sets of $G$ with size $k$ and $s(G, 0)=1$. A unicyclic graph is a graph containing exactly one cycle. Let $C_{n}$ be the cycle on $n$ vertices. In this paper we study the independence polynomial of unicyclic graphs. We show that among all connected unicyclic graphs $G$ on $n$ vertices (except two of them), $I(G, t)>I\left(C_{n}, t\right)$ for sufficiently large $t$. Finally for every $n \geq 3$ we find all connected graphs $H$ such that $I(H, x)=I\left(C_{n}, x\right)$.
\end{abstract}

Keywords: independence polynomial, independent set, unicyclic graphs.

2010 Mathematics Subject Classification: 05C30, 05C31, 05C38, 05C69.

\section{REFERENCES}

[1] S. Akbari, S. Alikhani, M.R. Oboudi and Y.H. Peng, On the zeros of domination polynomial of a graph, Combin. Graphs 531 (2010) 109-115. doi:10.1090/conm/531/10460

[2] S. Akbari and M.R. Oboudi, Cycles are determined by their domination polynomials, Ars Combin. 116 (2014) 353-358.

[3] S. Akbari and M.R. Oboudi, On the edge cover polynomial of a graph, European J. Combin. 34 (2013) 297-321. doi:10.1016/j.ejc.2012.05.005 
[4] S. Akbari, M.R. Oboudi and S. Qajar, On the rational independence roots, Combin. Graphs 531 (2010) 149-157.

doi:10.1090/conm/531/10464

[5] J.I. Brown, C.A. Hickman and R.J. Nowakowski, On the location of roots of independence polynomials, J. Algebraic Combin. 19 (2004) 273-282. doi:10.1023/B:JACO.0000030703.39946.70

[6] M. Chudnovsky and P. Seymour, The roots of the independence polynomial of a claw free graph, J. Combin. Theory Ser. B 97 (2007) 350-357. doi:10.1016/j.jctb.2006.06.001

[7] P. Csikvári and M.R. Oboudi, On the roots of edge cover polynomials of graphs, European J. Combin. 32 (2011) 1407-1416.

doi:10.1016/j.ejc.2011.06.009

[8] T. Derikvand and M.R. Oboudi, On the number of maximum independent sets of graphs, Trans. Combin. 3 (2014) 29-36.

[9] I. Gutman, Some analytical properties of the independence and matching polynomials, MATCH Commun. Math. Comput. Chem. 28 (1992) 139-150.

[10] I. Gutman and F. Harary, Generalizations of the matching polynomial, Util. Math. 24 (1983) 97-106.

[11] C. Hoede and X. Li, Clique polynomials and independent set polynomials of graphs, Discrete Math. 125 (1994) 219-228. doi:10.1016/0012-365X(94)90163-5

[12] T. Kotek, J. Preen and P. Tittmann, Domination polynomials of graph products. arXiv:1305.1475v2.

[13] V.E. Levit and E. Mandrescu, The independence polynomial of a graph-A survey, Proceedings of the 1st International Conference on Algebraic Informatics (Aristotle Univ. Thessaloniki, Thessaloniki, 2005) 233-254.

[14] J.A. Makowsky, E.V. Ravve and N.K. Blanchard, On the location of roots of graph polynomials, European J. Combin. 41 (2014) 1-19. doi:10.1016/j.ejc.2014.03.003

[15] M.R. Oboudi, On the largest real root of independence polynomials of trees, Ars Combin., to appear.

[16] M.R. Oboudi, On the roots of domination polynomial of graphs, Discrete Appl. Math. 205 (2016) 126-131. doi:10.1016/j.dam.2015.12.010

Received 14 September 2016

Revised 10 January 2017

Accepted 10 January 2017 